\title{
CONTENIDO POLÍNICO DE LA ATMÓSFERA DE MÁLAGA: AÑOS 1992 Y 1993.
}

\author{
Baltasar CABEZUDO, M.Mar TRIGO, Marta RECIO y Fco. Javier TORO
}

\begin{abstract}
RESUMEN. Análisis polínico del contenido de la atmósfera de Málaga: año 1992-1993. En el presente trabajo se hace un estudio de la incidencia que tuvo el polen en la atmósfera de Málaga durante los años 1992 y 1993, analizándose la evolución estacional de la cantidad y calidad del contenido de la misma. Igualmente, se hace un balance de la importacia relativa de los distintos pólenes a lo largo del año, a la vez que se indican cuáles fueron los días y las semanas de máxima concentración durante los dos años objeto de estudio.
\end{abstract}

Palabras clave. Aerobiología, polen, Málaga, sur de España.

ABSTRACT: Pollen analysis content in the atmosphere of Malaga: 1992 and 1993. During 1992 and 1993 we have analysed the pollen content in the atmosphere of Malaga (southern Spain). The sampling was carried out with the aid of a Burkard spore-trap located on the roof of the Faculty of Medicine, Malaga University, about $15 \mathrm{~m}$ above ground level. The pollen data, expressed in grains per cubic metre of air, were obtained by counting four lengths of the slide with a $40 \mathrm{X}$ objective $(0,45 \mathrm{~mm}$ field). During these years, we have observed that about $85 \%$ of total pollen was collected from February to June, with a maximum peak in May, while lowest monthly concentration was detected during January and from August to December. Along this period, the most important taxa in the atmosphere of Malaga were Cupressaceae and Olea europaea followed by Quercus, Chenopodiaceae-Amaranthaceae, Poaceae, Plantago, Urticaceae and Pinus, by importance order. So, the different peaks which occur along the year are fundamentally due to Cupressaceae in February; Cupressaceae, Pinus and Platanus in March; Quercus in April; Olea, Chenopodiaceae-Amaranthaceae and Poaceae in May and Casuarina in October.

Key words. Aerobiology, pollen, Malaga, southern Spain.

\section{INTRODUCCIÓN}

Durante los diez últimos años, se ha venido observando en Europa, especialmente en áreas urbanas, un incremento del número de personas que presenta sensibilidad a algún tipo de polen (D'Amato \& Spieksma, 1990), de tal forma que el estudio de la calidad y cantidad del contenido polínico de la atmósfera en estas zonas se ha convertido en una necesidad a la hora de establecer el agente causante de muchos trastornos de origen alérgico tales como conjuntivitis, rinitis o asma. 
La ciudad de Málaga, situada en el sur de España, presenta un clima típicamente mediterráneo, en donde las más importantes causas de polinosis son el polen de olivo, de gramíneas y de Parietaria (Burgos, 1991). Como en toda zona urbana, los aspectos más significativos de la flora son el deterioro de la vegetación natural, la existencia de amplias zonas de cultivos agrícolas, un importante componente de florá ornamental y aumento de hábitats ruderalizados. Destacamos por su importancia los cultivos de olivo, cítricos, almendro, vid, caña de azúcar, así como pequeñas huertas. La vegetación natural ha quedado relegada a algunos enclaves de alcornocales, encinares, coscojares y a sus estapas de degradación como jarales y tomillares. En muchos casos esta vegetación natural ha sido reemplazada por repoblaciones de pinos que favorecen la existencia de importantes zonas de pastizal.

Hasta el momento, sólo habían sido publicados algunos datos parciales sobre la incidencia de pólenes en Málaga, obtenidos por el Servicio de Alergología de la Residencia Sanitaria Carlos de Haya de Málaga durante los años 1982 y 1983 (Chaparro y Conde, 1984) y que han servido de base a trabajos más generales de aerobiología en España. Con el presente estudio se pretende hacer un balance de la incidencia que los distintos pólenes han tenido en la atmósfera de la ciudad a lo largo de los años 1992 y 1993 como avance a la realización del calendario polínico de la misma.

\section{MATERIAL Y MÉTODOS}

El muestreo se realizó utilizando un captador volumétrico tipo Burkard colocado en el tejado de la Facultad de Medicina de la Universidad de Málaga, a unos $15 \mathrm{~m}$ aproximadamente del nivel del suelo. Dicho edificio se encuentra situado a $1 \mathrm{Km}$ al oeste de la ciudad en una zona abierta, sin edificios colindantes que impidan la libre circulación del aire. En sus alrededores crece fundamentalmente una vegetación de tipo ruderal junto con algunas especies cultivadas como ornamentales, lo que hace que la diversidad del polen detectado sea bastente grande, razón por la cual en las tablas sólo se han incluido aquéllos taxones que en algún momento llegaron a superar el valor de 1 grano de polen $/ \mathrm{m}^{3}$ de aire como promedio semanal.

Para llevar a cabo el recuento de los distintos tipos polínicos se han realizado cuatro barridos por preparación, utilizándose un objetivo de 40 aumentos y extrapolándose posteriormente los resultados obtenidos al total de la muestra, según la metodología propuesta por Domínguez et al. (1991) para la Red Española de Aerobiología (R.E.A.). Como material adhesivo se utilizó petrolato blanco y como medio de montaje glicerogelatina. Los datos se expresan en número de granos de polen por metro cúbico de aire y hora, habiéndose utilizado los valores medios diarios para la confección de tablas y gráficas.

Los datos meteorológicos han sido faciltados por la estación Málaga-Aeropuerto a través del Centro Meteorológico Zonal de Málaga. Dicha estación se encuentra situada a $5 \mathrm{Km}$ de distancia de la estación de muestreo siendo, por otra parte, la más cercana a la misma.

\section{RESULTADOS}

En las tablas 1, 2 y 3 se presentan los valores totales mensuales y anuales, absolutos y relativos, de las concentraciones de polen de los distintos taxones estudiados, así como de la concentración de polen total durante los años 1992 y 1993.

Durante estos años, se observa que las mayores concentraciones de polen se produjeron durante los meses de Febrero a Junio, ambos inclusive, período en el que se registraron el $84,3 \%$ del total anual en el año 1992 y el $87,9 \%$ en el año 1993, con picos máximos 


\begin{tabular}{lcccc}
\hline Meses & Total & Total & $\%$ & $\%$ \\
& 1992 & 1993 & 1992 & 1993 \\
\hline Enero & 398 & 294 & 1,8 & 0,9 \\
Febrero & 4298 & 2660 & 19,1 & 8,4 \\
Marzo & 2440 & 3154 & 10,8 & 10 \\
Abril & 4620 & 5562 & 20,5 & 17,6 \\
Mayo & 6234 & 10166 & 27,7 & 32,2 \\
Junio & 1402 & 6219 & 6,2 & 19,7 \\
Julio & 792 & 1053 & 3,5 & 3,3 \\
Agosto & 506 & 467 & 2,2 & 1,5 \\
Septiembre & 472 & 426 & 2,1 & 1,3 \\
Octubre & 484 & 879 & 2,2 & 2,8 \\
Noviembre & 393 & 347 & 1,7 & 1,1 \\
Diciembre & 483 & 384 & 2,2 & 1,2 \\
\hline Total anual & 22522 & 31631 & 100 & 100 \\
\hline
\end{tabular}

Tabla 1. Valores mensuales absolutos $\left(\right.$ granos $\left./ \mathrm{m}^{3}\right) \mathrm{y}$ relativos (\%) obtenidos por las concentraciones de polen total a lo largo de los años 1992 y 1993. Monthly values (grains $/ \mathrm{m}^{3}$ ) and percentages (\%) obtained by the total pollen counts along the years 1992 and 1993.

durante el mes de Mayo. Por el contrario, las cantidades de polen registradas desde Julio a Diciembre, así como durante el mes de Enero, fueron bastante bajas, no superándose en ningún caso el nivel del 3,3\% del total anual (tab. 1, fig. 1).

Se observa que los taxones que mayor incidencia tuvieron en la atmósfera de Málaga durante el año 1992 fueron Cupresáceas $(21,7 \%$ del total anual) y olivo (19,9\%), mientras que durante 1993 el olivo alcanzó el 28,2\% y las Cupresáceas el 13,7\%. En orden de importancia les siguen Quercus, QuenopodiáceasAmarantáceas, Gramíneas, Plantago, Urticáceas y Pinus (tab. 3).

Los distintos picos que se suceden a lo largo del año (fig. 1) reflejan diferentes situaciones en el contenido polínico de la atmósfera. Así, mientras que durante el mes de Enero las concentraciones se mantienen en sus valo- res más bajos, durante el mes de Febrero se produce un primer pico debido en casi un $90 \%$ al polen de Cupresáceas, obsevándose un desfase de entre los valores máximos obtenidos durante los dos años de estudio (tab. 4).

Durante el mes de Marzo la situación es diferente, ya que las cantidades de polen de Cupresáceas se reducen considerablemente mientras que comienzan a aumentar las concentraciones de otros pólenes entre los que caben destacar Pinus, Platanus y Urticáceas, alcanzando Pinus y Platanus sus valores máximos durante este mes.

Abril se caracteriza por un nuevo incremento de las concentraciones de polen, si bien los niveles alcanzados se encuentran ampliamente influenciados por el régimen de lluvias así como por el comportamiento de las temperaturas durante esta época del año (figs. 2 y 3 ). En general, el incremento de las concentraciones se debe en gran medida al aumento de polen de Quercus, que alcanzó en este mes sus valores máximos, pero también a la aportación de otras especies como Plantago y Quenopodiáceas (especialmente Betavulgaris, muy frecuente en la zona) e incluso al olivo, que comienza a florecer por estas fechas.

El pico de Mayo se debe fundamentalmente al fuerte incremento que sufren las concentraciones de polen de olivo, que llegaron a suponer el $47,6 \%$ del total mensual durante 1992 y el $59,7 \%$ en 1993 , siguiéndole en importancia Quenopodiáceas, Gramíneas, Quercus y Plantago, aunque con valores de distinta intensidad de un año a otro. Mayo, por otra parte, es el mes en el que más tipos polínicos encontramos, alcanzando sus valores máximos especies como Rumex y Cyperáceas.

Durante el mes de Junio las concentraciones de polen se reducen considerablemente, aunque el de olivo aún se mantiene presente en la atmósfera en cantidades importantes y el de gramíneas aún puede alcanzar sus valores más altos, especialmente durante la primera sema- 


\begin{tabular}{|c|c|c|c|c|c|c|c|c|c|c|c|c|c|c|}
\hline Taxón & Año & Ene & Feb & Mar & $\mathrm{Abr}$ & May & Jun & Jul & Ago & Sep & Oct & Nov & Dic & Total anual \\
\hline Apiaceae & \begin{tabular}{|l|}
1992 \\
1993 \\
\end{tabular} & $\begin{array}{l}0 \\
0\end{array}$ & $\begin{array}{l}0 \\
2\end{array}$ & $\begin{array}{l}0 \\
1\end{array}$ & $\begin{array}{l}2 \\
6\end{array}$ & $\begin{array}{l}23 \\
12\end{array}$ & $\begin{array}{l}43 \\
52\end{array}$ & $\begin{array}{r}3 \\
14\end{array}$ & $\begin{array}{l}4 \\
5\end{array}$ & $\begin{array}{r}4 \\
12\end{array}$ & $\begin{array}{l}6 \\
4\end{array}$ & $\begin{array}{l}2 \\
0\end{array}$ & $\begin{array}{l}0 \\
1\end{array}$ & $\begin{array}{c}87 \\
109\end{array}$ \\
\hline Artemisia & $\begin{array}{l}1992 \\
1993\end{array}$ & $\begin{array}{l}53 \\
64\end{array}$ & $\begin{array}{l}18 \\
64\end{array}$ & $\begin{array}{l}1 \\
3\end{array}$ & $\begin{array}{l}0 \\
0\end{array}$ & $\begin{array}{l}0 \\
0\end{array}$ & $\begin{array}{l}0 \\
0\end{array}$ & $\begin{array}{l}4 \\
0\end{array}$ & $\begin{array}{r}21 \\
3\end{array}$ & $\begin{array}{r}79 \\
4\end{array}$ & $\begin{array}{l}1 \\
3\end{array}$ & $\begin{array}{r}42 \\
4\end{array}$ & $\begin{array}{r}310 \\
45\end{array}$ & $\begin{array}{l}529 \\
190\end{array}$ \\
\hline Castanea & $\begin{array}{l}1992 \\
1993\end{array}$ & $\begin{array}{l}0 \\
0\end{array}$ & $\begin{array}{l}0 \\
0\end{array}$ & $\begin{array}{l}1 \\
0\end{array}$ & $\begin{array}{l}1 \\
0\end{array}$ & $\begin{array}{l}0 \\
0\end{array}$ & $\begin{array}{l}24 \\
26\end{array}$ & $\begin{array}{r}5 \\
26\end{array}$ & $\begin{array}{l}1 \\
3\end{array}$ & $\begin{array}{l}1 \\
2\end{array}$ & $\begin{array}{l}0 \\
0\end{array}$ & $\begin{array}{l}0 \\
0\end{array}$ & $\begin{array}{l}0 \\
0\end{array}$ & $\begin{array}{l}33 \\
57\end{array}$ \\
\hline Casuarina & $\begin{array}{l}1992 \\
1993\end{array}$ & $\begin{array}{l}1 \\
1\end{array}$ & $\begin{array}{l}1 \\
0\end{array}$ & $\begin{array}{l}2 \\
0\end{array}$ & $\begin{array}{l}0 \\
0\end{array}$ & $\begin{array}{l}0 \\
0\end{array}$ & $\begin{array}{l}0 \\
0\end{array}$ & $\begin{array}{l}0 \\
0\end{array}$ & $\begin{array}{l}0 \\
0\end{array}$ & $\begin{array}{r}30 \\
1\end{array}$ & $\begin{array}{l}199 \\
691\end{array}$ & $\begin{array}{l}39 \\
84\end{array}$ & $\begin{array}{l}5 \\
9\end{array}$ & $\begin{array}{l}277 \\
786\end{array}$ \\
\hline $\begin{array}{l}\text { Compositae } \\
\text { (excl. Artemisia) }\end{array}$ & $\begin{array}{l}1992 \\
1993\end{array}$ & $\begin{array}{r}5 \\
11\end{array}$ & $\begin{array}{l}3 \\
2\end{array}$ & $\begin{array}{r}21 \\
7\end{array}$ & $\begin{array}{l}24 \\
28\end{array}$ & $\begin{array}{l}52 \\
33\end{array}$ & $\begin{array}{l}27 \\
47\end{array}$ & $\begin{array}{l}11 \\
34\end{array}$ & $\begin{array}{l}29 \\
23\end{array}$ & $\begin{array}{l}25 \\
25\end{array}$ & $\begin{array}{l}25 \\
11\end{array}$ & $\begin{array}{r}7 \\
14\end{array}$ & $\begin{array}{l}4 \\
3\end{array}$ & $\begin{array}{l}233 \\
238\end{array}$ \\
\hline Cupressaceae & $\begin{array}{l}1992 \\
1993\end{array}$ & $\begin{array}{l}65 \\
68\end{array}$ & $\begin{array}{l}3863 \\
2358\end{array}$ & $\begin{array}{r}567 \\
1654\end{array}$ & $\begin{array}{l}35 \\
73\end{array}$ & $\begin{array}{r}108 \\
9\end{array}$ & $\begin{array}{r}4 \\
24\end{array}$ & $\begin{array}{r}21 \\
5\end{array}$ & $\begin{array}{l}2 \\
2\end{array}$ & $\begin{array}{l}1 \\
1\end{array}$ & $\begin{array}{l}70 \\
27\end{array}$ & $\begin{array}{r}130 \\
61\end{array}$ & $\begin{array}{l}22 \\
65\end{array}$ & $\begin{array}{l}4888 \\
4347\end{array}$ \\
\hline Cyperaceae & $\begin{array}{l}1992 \\
1993\end{array}$ & $\begin{array}{l}0 \\
0\end{array}$ & $\begin{array}{l}0 \\
0\end{array}$ & $\begin{array}{l}0 \\
2\end{array}$ & $\begin{array}{l}4 \\
8\end{array}$ & $\begin{array}{l}129 \\
100\end{array}$ & $\begin{array}{l}70 \\
83\end{array}$ & $\begin{array}{l}18 \\
22\end{array}$ & $\begin{array}{l}5 \\
4\end{array}$ & $\begin{array}{l}1 \\
1\end{array}$ & $\begin{array}{l}0 \\
0\end{array}$ & $\begin{array}{l}0 \\
1\end{array}$ & $\begin{array}{l}1 \\
0\end{array}$ & $\begin{array}{l}228 \\
221\end{array}$ \\
\hline $\begin{array}{l}\text { Chenopodiaceae y } \\
\text { Amaranthaceae }\end{array}$ & $\begin{array}{l}1992 \\
1993\end{array}$ & $\begin{array}{l}3 \\
5\end{array}$ & $\begin{array}{r}10 \\
7\end{array}$ & $\begin{array}{l}15 \\
15\end{array}$ & $\begin{array}{l}483 \\
755\end{array}$ & $\begin{array}{r}464 \\
1056\end{array}$ & $\begin{array}{r}86 \\
305\end{array}$ & $\begin{array}{r}95 \\
106\end{array}$ & $\begin{array}{l}130 \\
120\end{array}$ & $\begin{array}{l}163 \\
147\end{array}$ & $\begin{array}{l}27 \\
19\end{array}$ & $\begin{array}{l}9 \\
2\end{array}$ & $\begin{array}{l}7 \\
2\end{array}$ & $\begin{array}{l}1492 \\
2539\end{array}$ \\
\hline Echium & $\begin{array}{l}1992 \\
1993\end{array}$ & $\begin{array}{l}0 \\
0\end{array}$ & $\begin{array}{l}1 \\
1\end{array}$ & $\begin{array}{r}6 \\
13\end{array}$ & $\begin{array}{l}21 \\
55\end{array}$ & $\begin{array}{l}11 \\
40\end{array}$ & $\begin{array}{r}6 \\
14\end{array}$ & $\begin{array}{l}1 \\
1\end{array}$ & $\begin{array}{l}0 \\
6\end{array}$ & $\begin{array}{l}0 \\
0\end{array}$ & $\begin{array}{l}1 \\
0\end{array}$ & $\begin{array}{l}0 \\
0\end{array}$ & $\begin{array}{l}0 \\
0\end{array}$ & $\begin{array}{c}47 \\
130\end{array}$ \\
\hline Ericaceae & $\begin{array}{l}1992 \\
1993\end{array}$ & $\begin{array}{l}0 \\
0\end{array}$ & $\begin{array}{l}0 \\
2\end{array}$ & $\begin{array}{r}5 \\
18\end{array}$ & $\begin{array}{l}14 \\
13\end{array}$ & $\begin{array}{r}9 \\
86\end{array}$ & $\begin{array}{r}2 \\
16\end{array}$ & $\begin{array}{l}0 \\
0\end{array}$ & $\begin{array}{l}0 \\
0\end{array}$ & $\begin{array}{l}0 \\
1\end{array}$ & $\begin{array}{l}1 \\
1\end{array}$ & $\begin{array}{l}0 \\
1\end{array}$ & $\begin{array}{l}0 \\
1\end{array}$ & $\begin{array}{c}31 \\
139\end{array}$ \\
\hline Gramineae & $\begin{array}{l}1992 \\
1993\end{array}$ & $\begin{array}{r}31 \\
9\end{array}$ & $\begin{array}{r}12 \\
6\end{array}$ & $\begin{array}{l}94 \\
22\end{array}$ & $\begin{array}{r}188 \\
53\end{array}$ & $\begin{array}{l}491 \\
537\end{array}$ & $\begin{array}{l}163 \\
733\end{array}$ & $\begin{array}{l}122 \\
118\end{array}$ & $\begin{array}{l}73 \\
46\end{array}$ & $\begin{array}{l}13 \\
47\end{array}$ & $\begin{array}{l}31 \\
11\end{array}$ & $\begin{array}{r}25 \\
2\end{array}$ & $\begin{array}{r}6 \\
11\end{array}$ & $\begin{array}{l}1249 \\
1595\end{array}$ \\
\hline Mercurialis & $\begin{array}{l}1992 \\
1993\end{array}$ & $\begin{array}{l}47 \\
12\end{array}$ & $\begin{array}{l}35 \\
35\end{array}$ & $\begin{array}{r}73 \\
115\end{array}$ & $\begin{array}{l}51 \\
56\end{array}$ & $\begin{array}{l}4 \\
3\end{array}$ & $\begin{array}{l}4 \\
4\end{array}$ & $\begin{array}{l}3 \\
1\end{array}$ & $\begin{array}{l}0 \\
1\end{array}$ & $\begin{array}{l}0 \\
1\end{array}$ & $\begin{array}{l}3 \\
2\end{array}$ & $\begin{array}{l}3 \\
2\end{array}$ & $\begin{array}{r}8 \\
11\end{array}$ & $\begin{array}{l}231 \\
243\end{array}$ \\
\hline Morus & $\begin{array}{l}1992 \\
1993 \\
\end{array}$ & $\begin{array}{l}1 \\
0\end{array}$ & $\begin{array}{l}2 \\
1\end{array}$ & $\begin{array}{l}35 \\
12\end{array}$ & $\begin{array}{l}15 \\
24\end{array}$ & $\begin{array}{l}5 \\
2\end{array}$ & $\begin{array}{l}1 \\
0\end{array}$ & $\begin{array}{l}1 \\
0\end{array}$ & $\begin{array}{l}0 \\
0\end{array}$ & $\begin{array}{l}0 \\
0\end{array}$ & $\begin{array}{l}0 \\
0\end{array}$ & $\begin{array}{l}0 \\
0\end{array}$ & $\begin{array}{l}1 \\
0\end{array}$ & $\begin{array}{l}61 \\
39\end{array}$ \\
\hline Myrtaceae & \begin{tabular}{|l|}
1992 \\
1993
\end{tabular} & $\begin{array}{l}0 \\
4\end{array}$ & $\begin{array}{l}0 \\
1\end{array}$ & $\begin{array}{l}3 \\
3\end{array}$ & $\begin{array}{l}2 \\
6\end{array}$ & $\begin{array}{l}28 \\
17\end{array}$ & $\begin{array}{l}317 \\
157\end{array}$ & $\begin{array}{l}102 \\
228\end{array}$ & $\begin{array}{l}35 \\
51\end{array}$ & $\begin{array}{l}29 \\
27\end{array}$ & $\begin{array}{l}10 \\
13\end{array}$ & $\begin{array}{l}1 \\
3\end{array}$ & $\begin{array}{l}6 \\
2\end{array}$ & $\begin{array}{l}533 \\
512\end{array}$ \\
\hline Olea & $\begin{array}{l}1992 \\
1993\end{array}$ & $\begin{array}{l}0 \\
1\end{array}$ & $\begin{array}{l}0 \\
1\end{array}$ & $\begin{array}{r}24 \\
6\end{array}$ & $\begin{array}{r}1292 \\
236\end{array}$ & $\begin{array}{l}2966 \\
6081\end{array}$ & $\begin{array}{r}161 \\
2488\end{array}$ & $\begin{array}{l}20 \\
84\end{array}$ & $\begin{array}{r}6 \\
13\end{array}$ & $\begin{array}{l}2 \\
8\end{array}$ & $\begin{array}{l}1 \\
4\end{array}$ & $\begin{array}{l}7 \\
1\end{array}$ & $\begin{array}{l}2 \\
1\end{array}$ & $\begin{array}{l}4481 \\
8924\end{array}$ \\
\hline Palmae & $\begin{array}{l}1992 \\
1993\end{array}$ & $\begin{array}{l}25 \\
10\end{array}$ & $\begin{array}{l}7 \\
1\end{array}$ & $\begin{array}{l}3 \\
4\end{array}$ & $\begin{array}{l}6 \\
6\end{array}$ & $\begin{array}{l}1 \\
2\end{array}$ & $\begin{array}{l}0 \\
1\end{array}$ & $\begin{array}{r}20 \\
4\end{array}$ & $\begin{array}{r}17 \\
5\end{array}$ & $\begin{array}{r}44 \\
6\end{array}$ & $\begin{array}{r}23 \\
2\end{array}$ & $\begin{array}{l}17 \\
55\end{array}$ & $\begin{array}{r}17 \\
3\end{array}$ & $\begin{array}{c}180 \\
99\end{array}$ \\
\hline Parkinsonia & \begin{tabular}{|l|}
1992 \\
1993
\end{tabular} & $\begin{array}{l}0 \\
0\end{array}$ & $\begin{array}{l}0 \\
0\end{array}$ & $\begin{array}{l}0 \\
0\end{array}$ & $\begin{array}{l}5 \\
0\end{array}$ & $\begin{array}{l}3 \\
0\end{array}$ & $\begin{array}{l}42 \\
42\end{array}$ & $\begin{array}{l}14 \\
21\end{array}$ & $\begin{array}{r}12 \\
4\end{array}$ & $\begin{array}{l}1 \\
4\end{array}$ & $\begin{array}{l}1 \\
0\end{array}$ & $\begin{array}{l}0 \\
0\end{array}$ & $\begin{array}{l}0 \\
0\end{array}$ & $\begin{array}{l}78 \\
71\end{array}$ \\
\hline Pinus & $\begin{array}{l}1992 \\
1993\end{array}$ & $\begin{array}{l}0 \\
0\end{array}$ & $\begin{array}{r}100 \\
32\end{array}$ & $\begin{array}{l}323 \\
452\end{array}$ & $\begin{array}{l}104 \\
243\end{array}$ & $\begin{array}{r}52 \\
108\end{array}$ & $\begin{array}{r}14 \\
415\end{array}$ & $\begin{array}{l}35 \\
25\end{array}$ & $\begin{array}{l}3 \\
6\end{array}$ & $\begin{array}{l}1 \\
3\end{array}$ & $\begin{array}{l}1 \\
1\end{array}$ & $\begin{array}{l}0 \\
0\end{array}$ & $\begin{array}{l}0 \\
0\end{array}$ & $\begin{array}{c}633 \\
1285\end{array}$ \\
\hline Pistacia & \begin{tabular}{|l|}
1992 \\
1993
\end{tabular} & $\begin{array}{l}0 \\
0\end{array}$ & $\begin{array}{l}0 \\
0\end{array}$ & $\begin{array}{l}36 \\
18\end{array}$ & $\begin{array}{l}35 \\
78\end{array}$ & $\begin{array}{l}2 \\
4\end{array}$ & $\begin{array}{l}0 \\
0\end{array}$ & $\begin{array}{l}0 \\
0\end{array}$ & $\begin{array}{l}0 \\
0\end{array}$ & $\begin{array}{l}0 \\
0\end{array}$ & $\begin{array}{l}0 \\
0\end{array}$ & $\begin{array}{l}0 \\
0\end{array}$ & $\begin{array}{l}0 \\
0\end{array}$ & $\begin{array}{c}73 \\
100\end{array}$ \\
\hline Plantago & $\begin{array}{l}1992 \\
1993\end{array}$ & $\begin{array}{l}0 \\
1\end{array}$ & $\begin{array}{l}0 \\
2\end{array}$ & $\begin{array}{r}103 \\
57\end{array}$ & $\begin{array}{l}535 \\
523\end{array}$ & $\begin{array}{l}302 \\
634\end{array}$ & $\begin{array}{r}75 \\
250\end{array}$ & $\begin{array}{l}66 \\
39\end{array}$ & $\begin{array}{l}10 \\
10\end{array}$ & $\begin{array}{l}6 \\
4\end{array}$ & $\begin{array}{l}5 \\
0\end{array}$ & $\begin{array}{l}1 \\
1\end{array}$ & $\begin{array}{l}1 \\
0\end{array}$ & $\begin{array}{l}1104 \\
1521\end{array}$ \\
\hline Platanus & $\begin{array}{l}1992 \\
1993\end{array}$ & $\begin{array}{l}0 \\
0\end{array}$ & $\begin{array}{l}0 \\
0\end{array}$ & $\begin{array}{l}181 \\
246\end{array}$ & $\begin{array}{l}16 \\
31\end{array}$ & $\begin{array}{l}0 \\
1\end{array}$ & $\begin{array}{l}0 \\
0\end{array}$ & $\begin{array}{l}0 \\
0\end{array}$ & $\begin{array}{l}0 \\
0\end{array}$ & $\begin{array}{l}0 \\
0\end{array}$ & $\begin{array}{l}0 \\
0\end{array}$ & $\begin{array}{l}0 \\
0\end{array}$ & $\begin{array}{l}0 \\
0\end{array}$ & $\begin{array}{l}197 \\
278\end{array}$ \\
\hline Populus & \begin{tabular}{|l|}
1992 \\
1993
\end{tabular} & $\begin{array}{l}0 \\
0\end{array}$ & $\begin{array}{l}45 \\
11\end{array}$ & $\begin{array}{l}75 \\
25\end{array}$ & $\begin{array}{l}1 \\
0\end{array}$ & $\begin{array}{l}0 \\
0\end{array}$ & $\begin{array}{l}0 \\
0\end{array}$ & $\begin{array}{l}0 \\
0\end{array}$ & $\begin{array}{l}0 \\
0\end{array}$ & $\begin{array}{l}0 \\
0\end{array}$ & $\begin{array}{l}0 \\
0\end{array}$ & $\begin{array}{l}0 \\
0\end{array}$ & $\begin{array}{l}0 \\
0\end{array}$ & $\begin{array}{c}121 \\
36\end{array}$ \\
\hline Quercus & $\begin{array}{l}1992 \\
1993\end{array}$ & $\begin{array}{l}0 \\
2\end{array}$ & $\begin{array}{l}0 \\
0\end{array}$ & $\begin{array}{r}200 \\
47\end{array}$ & $\begin{array}{r}904 \\
2146\end{array}$ & $\begin{array}{l}736 \\
460\end{array}$ & $\begin{array}{r}65 \\
377\end{array}$ & $\begin{array}{l}11 \\
20\end{array}$ & $\begin{array}{l}2 \\
4\end{array}$ & $\begin{array}{l}1 \\
7\end{array}$ & $\begin{array}{l}2 \\
5\end{array}$ & $\begin{array}{l}1 \\
1\end{array}$ & $\begin{array}{l}3 \\
2\end{array}$ & $\begin{array}{l}1925 \\
3071\end{array}$ \\
\hline Ricinus & \begin{tabular}{|l|}
1992 \\
1993
\end{tabular} & $\begin{array}{r}27 \\
4\end{array}$ & $\begin{array}{l}7 \\
2\end{array}$ & $\begin{array}{r}14 \\
4\end{array}$ & $\begin{array}{r}17 \\
5\end{array}$ & $\begin{array}{r}28 \\
8\end{array}$ & $\begin{array}{l}4 \\
2\end{array}$ & $\begin{array}{l}8 \\
1\end{array}$ & $\begin{array}{l}7 \\
2\end{array}$ & $\begin{array}{l}2 \\
3\end{array}$ & $\begin{array}{l}2 \\
1\end{array}$ & $\begin{array}{r}12 \\
4\end{array}$ & $\begin{array}{r}11 \\
6\end{array}$ & $\begin{array}{c}139 \\
42\end{array}$ \\
\hline Rumex & \begin{tabular}{|l|}
1992 \\
1993
\end{tabular} & $\begin{array}{l}1 \\
2\end{array}$ & $\begin{array}{l}0 \\
3\end{array}$ & $\begin{array}{l}41 \\
26\end{array}$ & $\begin{array}{l}122 \\
110\end{array}$ & $\begin{array}{r}83 \\
136\end{array}$ & $\begin{array}{l}21 \\
74\end{array}$ & $\begin{array}{r}8 \\
11\end{array}$ & $\begin{array}{l}1 \\
1\end{array}$ & $\begin{array}{l}0 \\
1\end{array}$ & $\begin{array}{l}0 \\
0\end{array}$ & $\begin{array}{l}1 \\
0\end{array}$ & $\begin{array}{l}1 \\
0\end{array}$ & $\begin{array}{l}279 \\
364\end{array}$ \\
\hline Typha & \begin{tabular}{|l|}
1992 \\
1993
\end{tabular} & $\begin{array}{l}0 \\
0\end{array}$ & $\begin{array}{l}0 \\
0\end{array}$ & $\begin{array}{l}0 \\
0\end{array}$ & $\begin{array}{l}0 \\
0\end{array}$ & $\begin{array}{r}12 \\
0\end{array}$ & $\begin{array}{l}36 \\
38\end{array}$ & $\begin{array}{l}3 \\
4\end{array}$ & $\begin{array}{l}1 \\
2\end{array}$ & $\begin{array}{l}1 \\
1\end{array}$ & $\begin{array}{l}0 \\
0\end{array}$ & $\begin{array}{l}0 \\
0\end{array}$ & $\begin{array}{l}0 \\
0\end{array}$ & $\begin{array}{l}53 \\
45\end{array}$ \\
\hline Ulmus & \begin{tabular}{|l|}
1992 \\
1993
\end{tabular} & $\begin{array}{l}1 \\
0\end{array}$ & $\begin{array}{l}7 \\
4\end{array}$ & $\begin{array}{l}2 \\
7\end{array}$ & $\begin{array}{l}0 \\
0\end{array}$ & $\begin{array}{l}0 \\
0\end{array}$ & $\begin{array}{l}0 \\
0\end{array}$ & $\begin{array}{l}0 \\
0\end{array}$ & $\begin{array}{l}0 \\
0\end{array}$ & $\begin{array}{l}0 \\
0\end{array}$ & $\begin{array}{l}0 \\
0\end{array}$ & $\begin{array}{l}0 \\
0\end{array}$ & $\begin{array}{l}0 \\
0\end{array}$ & $\begin{array}{l}10 \\
11\end{array}$ \\
\hline Urticaceae & \begin{tabular}{|l|}
1992 \\
1993
\end{tabular} & $\begin{array}{l}48 \\
63\end{array}$ & $\begin{array}{l}98 \\
78\end{array}$ & $\begin{array}{l}182 \\
213\end{array}$ & $\begin{array}{l}216 \\
269\end{array}$ & $\begin{array}{l}122 \\
197\end{array}$ & $\begin{array}{r}83 \\
146\end{array}$ & $\begin{array}{l}62 \\
45\end{array}$ & $\begin{array}{r}9 \\
17\end{array}$ & $\begin{array}{l}3 \\
7\end{array}$ & $\begin{array}{l}7 \\
5\end{array}$ & $\begin{array}{l}43 \\
67\end{array}$ & $\begin{array}{r}33 \\
135\end{array}$ & $\begin{array}{c}906 \\
1242\end{array}$ \\
\hline Otros & $\begin{array}{l}1992 \\
1993 \\
\end{array}$ & $\begin{array}{l}90 \\
37\end{array}$ & $\begin{array}{l}89 \\
47\end{array}$ & $\begin{array}{l}433 \\
184\end{array}$ & $\begin{array}{l}527 \\
838\end{array}$ & $\begin{array}{l}603 \\
660\end{array}$ & $\begin{array}{l}154 \\
925\end{array}$ & $\begin{array}{l}159 \\
244\end{array}$ & $\begin{array}{l}138 \\
139\end{array}$ & $\begin{array}{r}65 \\
113\end{array}$ & $\begin{array}{l}68 \\
79\end{array}$ & $\begin{array}{l}53 \\
44\end{array}$ & $\begin{array}{l}45 \\
87\end{array}$ & $\begin{array}{l}2424 \\
3397\end{array}$ \\
\hline
\end{tabular}

Tabla 2. Sumas mensuales y anuales de las concentraciones medias diarias de polen $\left(\mathrm{n}^{\circ}\right.$ granos $\left./ \mathrm{m}^{3}\right)$ obtenidas por los distintos táxones estudiados durante los años 1992 y 1993. Monthly and annual sum of the daily average concentrations (grains / $\mathrm{m}^{3}$ ) rise by the diferent taxa during 1992 and 1993. 


\begin{tabular}{|c|c|c|c|c|c|c|c|c|c|c|c|c|c|c|}
\hline Taxón & Año & \%Ene & $\%$ Feb & $\% \mathrm{Mar}$ & $\%$ Abr & \%May & \%Jun & \%Jul & $\%$ Ago & \%Sep & $\%$ Oct & \%Nov & \%Dic & \% Total anua \\
\hline Apiaceae & $\begin{array}{l}1992 \\
1993\end{array}$ & $\begin{array}{l}0,0 \\
0,0\end{array}$ & $\begin{array}{l}0,0 \\
0,1\end{array}$ & $\begin{array}{l}0,0 \\
0,0\end{array}$ & $\begin{array}{l}0,0 \\
0,1\end{array}$ & $\begin{array}{l}0,4 \\
0,1\end{array}$ & $\begin{array}{l}3,1 \\
0,8\end{array}$ & $\begin{array}{l}0,4 \\
1,3\end{array}$ & $\begin{array}{l}0,8 \\
1,1\end{array}$ & $\begin{array}{l}0,8 \\
2,8\end{array}$ & $\begin{array}{l}1,2 \\
0,5\end{array}$ & $\begin{array}{l}0,5 \\
0,0\end{array}$ & $\begin{array}{l}0,0 \\
0,3\end{array}$ & $\begin{array}{l}0,4 \\
0,3\end{array}$ \\
\hline Artemisia & $\begin{array}{l}1992 \\
1993\end{array}$ & $\begin{array}{l}13,3 \\
21,8\end{array}$ & $\begin{array}{l}0,4 \\
2,4\end{array}$ & $\begin{array}{l}0,0 \\
0,1\end{array}$ & $\begin{array}{l}0,0 \\
0,0\end{array}$ & $\begin{array}{l}0,0 \\
0,0\end{array}$ & $\begin{array}{l}0,0 \\
0,0\end{array}$ & $\begin{array}{l}0,5 \\
0,0\end{array}$ & $\begin{array}{l}4,2 \\
0,6\end{array}$ & $\begin{array}{r}16,7 \\
0,9\end{array}$ & $\begin{array}{l}0,2 \\
0,3\end{array}$ & $\begin{array}{r}10,7 \\
1,2\end{array}$ & $\begin{array}{l}64,2 \\
11,7\end{array}$ & $\begin{array}{l}2,3 \\
0,6\end{array}$ \\
\hline Castanea & $\begin{array}{l}1992 \\
1993\end{array}$ & $\begin{array}{l}0,0 \\
0,0\end{array}$ & $\begin{array}{l}0,0 \\
0,0\end{array}$ & $\begin{array}{l}0,0 \\
0,0\end{array}$ & $\begin{array}{l}0,0 \\
0,0\end{array}$ & $\begin{array}{l}0,0 \\
0,0\end{array}$ & $\begin{array}{l}1,7 \\
0,4\end{array}$ & $\begin{array}{l}0,6 \\
2,5\end{array}$ & $\begin{array}{l}0,2 \\
0,6\end{array}$ & $\begin{array}{l}0,2 \\
0,5\end{array}$ & $\begin{array}{l}0,0 \\
0,0\end{array}$ & $\begin{array}{l}0,0 \\
0,0\end{array}$ & $\begin{array}{l}0,0 \\
0,0\end{array}$ & $\begin{array}{l}0,1 \\
0,2\end{array}$ \\
\hline Casuarina & $\begin{array}{l}1992 \\
1993\end{array}$ & $\begin{array}{l}0,3 \\
0,3\end{array}$ & $\begin{array}{l}0,0 \\
0,0\end{array}$ & $\begin{array}{l}0,1 \\
0,0\end{array}$ & $\begin{array}{l}0,0 \\
0,0\end{array}$ & $\begin{array}{l}0,0 \\
0,0\end{array}$ & $\begin{array}{l}0,0 \\
0,0\end{array}$ & $\begin{array}{l}0,0 \\
0,0\end{array}$ & $\begin{array}{l}0,0 \\
0,0\end{array}$ & $\begin{array}{l}6,4 \\
0,2\end{array}$ & $\begin{array}{l}41,1 \\
78,6\end{array}$ & $\begin{array}{r}9,9 \\
24,2\end{array}$ & $\begin{array}{l}1,0 \\
2,3\end{array}$ & $\begin{array}{l}1,2 \\
2,5\end{array}$ \\
\hline $\begin{array}{l}\text { Compositae } \\
\text { (excl. Artemisia) }\end{array}$ & $\begin{array}{l}1992 \\
1993\end{array}$ & $\begin{array}{l}1,3 \\
3,7\end{array}$ & $\begin{array}{l}0,1 \\
0,1\end{array}$ & $\begin{array}{l}0,9 \\
0,2\end{array}$ & $\begin{array}{l}0,5 \\
0,5\end{array}$ & $\begin{array}{l}0,8 \\
0,3\end{array}$ & $\begin{array}{l}1,9 \\
0,8\end{array}$ & $\begin{array}{l}1,4 \\
3,2\end{array}$ & $\begin{array}{l}5,7 \\
4,9\end{array}$ & $\begin{array}{l}5,3 \\
5,9\end{array}$ & $\begin{array}{l}5,2 \\
1,3\end{array}$ & $\begin{array}{l}1,8 \\
4,0\end{array}$ & $\begin{array}{l}0,8 \\
0,8\end{array}$ & $\begin{array}{l}1,0 \\
0,8\end{array}$ \\
\hline Cupressaceae & $\begin{array}{l}1992 \\
1993\end{array}$ & $\begin{array}{l}16,3 \\
23,1\end{array}$ & $\begin{array}{l}89,9 \\
88,6\end{array}$ & $\begin{array}{l}23,2 \\
52,4\end{array}$ & $\begin{array}{l}0,8 \\
1,3\end{array}$ & $\begin{array}{l}1,7 \\
0,1\end{array}$ & $\begin{array}{l}0,3 \\
0,4\end{array}$ & $\begin{array}{l}2,7 \\
0,5\end{array}$ & $\begin{array}{l}0,4 \\
0,4\end{array}$ & $\begin{array}{l}0,2 \\
0,2\end{array}$ & $\begin{array}{r}14,5 \\
3,1\end{array}$ & $\begin{array}{l}33,1 \\
17,6\end{array}$ & $\begin{array}{r}4,6 \\
16,9\end{array}$ & $\begin{array}{l}21,7 \\
13,3\end{array}$ \\
\hline Cyperaceae & $\begin{array}{l}1992 \\
1993\end{array}$ & $\begin{array}{l}0,0 \\
0,0\end{array}$ & $\begin{array}{l}0,0 \\
0,0\end{array}$ & $\begin{array}{l}0,0 \\
0,1\end{array}$ & $\begin{array}{l}0,1 \\
0,1\end{array}$ & $\begin{array}{l}2,1 \\
1,0\end{array}$ & $\begin{array}{l}5,0 \\
1,3\end{array}$ & $\begin{array}{l}2,3 \\
2,1\end{array}$ & $\begin{array}{l}1,0 \\
0,9\end{array}$ & $\begin{array}{l}0,2 \\
0,2\end{array}$ & $\begin{array}{l}0,0 \\
0,0\end{array}$ & $\begin{array}{l}0,0 \\
0,3\end{array}$ & $\begin{array}{l}0,2 \\
0,0\end{array}$ & $\begin{array}{l}1,0 \\
0,7\end{array}$ \\
\hline $\begin{array}{l}\text { Chenopodiaceae y } \\
\text { Amaranthaceae }\end{array}$ & $\begin{array}{l}1992 \\
1993\end{array}$ & $\begin{array}{l}0,8 \\
1,7\end{array}$ & $\begin{array}{l}0,2 \\
0,3\end{array}$ & $\begin{array}{l}0,6 \\
0,5\end{array}$ & $\begin{array}{l}10,5 \\
13,6\end{array}$ & $\begin{array}{r}7,4 \\
10,4\end{array}$ & $\begin{array}{l}6,1 \\
4,9\end{array}$ & $\begin{array}{l}12,0 \\
10,1\end{array}$ & $\begin{array}{l}25,7 \\
25,7\end{array}$ & $\begin{array}{l}34,5 \\
34,5\end{array}$ & $\begin{array}{l}5,6 \\
2,2\end{array}$ & $\begin{array}{l}2,3 \\
0,6\end{array}$ & $\begin{array}{l}1,4 \\
0,5\end{array}$ & $\begin{array}{l}6,6 \\
8,0\end{array}$ \\
\hline Echium & $\begin{array}{l}1992 \\
1993\end{array}$ & $\begin{array}{l}0,0 \\
0,0\end{array}$ & $\begin{array}{l}0,0 \\
0,0\end{array}$ & $\begin{array}{l}0,2 \\
0,4\end{array}$ & $\begin{array}{l}0,5 \\
1,0\end{array}$ & $\begin{array}{l}0,2 \\
0,4\end{array}$ & $\begin{array}{l}0,4 \\
0,2\end{array}$ & $\begin{array}{l}0,1 \\
0,1\end{array}$ & $\begin{array}{l}0,0 \\
1,3\end{array}$ & $\begin{array}{l}0,0 \\
0,0\end{array}$ & $\begin{array}{l}0,2 \\
0,0\end{array}$ & $\begin{array}{l}0,0 \\
0,0\end{array}$ & $\begin{array}{l}0,0 \\
0,0\end{array}$ & $\begin{array}{l}0,2 \\
0,4\end{array}$ \\
\hline Ericaceae & $\begin{array}{l}1992 \\
1993\end{array}$ & $\begin{array}{l}0,0 \\
0,0\end{array}$ & $\begin{array}{l}0,0 \\
0,1\end{array}$ & $\begin{array}{l}0,2 \\
0,6\end{array}$ & $\begin{array}{l}0,3 \\
0,2\end{array}$ & $\begin{array}{l}0,1 \\
0,8\end{array}$ & $\begin{array}{l}0,1 \\
0,3\end{array}$ & $\begin{array}{l}0,0 \\
0,0\end{array}$ & $\begin{array}{l}0,0 \\
0,0\end{array}$ & $\begin{array}{l}0,0 \\
0,2\end{array}$ & $\begin{array}{l}0,2 \\
0,1\end{array}$ & $\begin{array}{l}0,0 \\
0,3\end{array}$ & $\begin{array}{l}0,0 \\
0,3\end{array}$ & $\begin{array}{l}0,1 \\
0,4\end{array}$ \\
\hline Gramineae & $\begin{array}{l}1992 \\
1993\end{array}$ & $\begin{array}{l}7,8 \\
3,1\end{array}$ & $\begin{array}{l}0,3 \\
0,2\end{array}$ & $\begin{array}{l}3,9 \\
0,7\end{array}$ & $\begin{array}{l}4,1 \\
1,0\end{array}$ & $\begin{array}{l}7,9 \\
5,3\end{array}$ & $\begin{array}{l}11,6 \\
11,8\end{array}$ & $\begin{array}{l}15,4 \\
11,2\end{array}$ & $\begin{array}{r}14,4 \\
9,9\end{array}$ & $\begin{array}{r}2,8 \\
11,0\end{array}$ & $\begin{array}{l}6,4 \\
1,3\end{array}$ & $\begin{array}{l}6,4 \\
0,6\end{array}$ & $\begin{array}{l}1,2 \\
2,9\end{array}$ & $\begin{array}{l}5,5 \\
5,0\end{array}$ \\
\hline Mercurialis & $\begin{array}{l}1992 \\
1993\end{array}$ & $\begin{array}{r}11,8 \\
4,1\end{array}$ & $\begin{array}{l}0,8 \\
1,3\end{array}$ & $\begin{array}{l}3,0 \\
3,6\end{array}$ & $\begin{array}{l}1,1 \\
1,0\end{array}$ & $\begin{array}{l}0,1 \\
0,0\end{array}$ & $\begin{array}{l}0,3 \\
0,1\end{array}$ & $\begin{array}{l}0,4 \\
0,1\end{array}$ & $\begin{array}{l}0,0 \\
0,2\end{array}$ & $\begin{array}{l}0,0 \\
0,2\end{array}$ & $\begin{array}{l}0,6 \\
0,2\end{array}$ & $\begin{array}{l}0,8 \\
0,6\end{array}$ & $\begin{array}{l}1,7 \\
2,9\end{array}$ & $\begin{array}{l}1,0 \\
0,8\end{array}$ \\
\hline Morus & $\begin{array}{l}1992 \\
1993\end{array}$ & $\begin{array}{l}0,3 \\
0,0\end{array}$ & $\begin{array}{l}0,0 \\
0,0\end{array}$ & $\begin{array}{l}1,4 \\
0,4\end{array}$ & $\begin{array}{l}0,3 \\
0,4\end{array}$ & $\begin{array}{l}0,1 \\
0,0\end{array}$ & $\begin{array}{l}0,1 \\
0,0\end{array}$ & $\begin{array}{l}0,1 \\
0,0\end{array}$ & $\begin{array}{l}0,0 \\
0,0\end{array}$ & $\begin{array}{l}0,0 \\
0,0\end{array}$ & $\begin{array}{l}0,0 \\
0,0\end{array}$ & $\begin{array}{l}0,0 \\
0,0\end{array}$ & $\begin{array}{l}0,2 \\
0,0\end{array}$ & $\begin{array}{l}0,3 \\
0,1\end{array}$ \\
\hline Myrtaceae & $\begin{array}{l}1992 \\
1993\end{array}$ & $\begin{array}{l}0,0 \\
1,4\end{array}$ & $\begin{array}{l}0,0 \\
0,0\end{array}$ & $\begin{array}{l}0,1 \\
0,1\end{array}$ & $\begin{array}{l}0,0 \\
0,1\end{array}$ & $\begin{array}{l}0,4 \\
0,2\end{array}$ & $\begin{array}{r}22,6 \\
2,5\end{array}$ & $\begin{array}{l}12,9 \\
21,7\end{array}$ & $\begin{array}{r}6,9 \\
10,9\end{array}$ & $\begin{array}{l}6,1 \\
6,3\end{array}$ & $\begin{array}{l}2,1 \\
1,5\end{array}$ & $\begin{array}{l}0,3 \\
0,9\end{array}$ & $\begin{array}{l}1,2 \\
0,5\end{array}$ & $\begin{array}{l}2,4 \\
1,6\end{array}$ \\
\hline Olea & $\begin{array}{l}1992 \\
1993\end{array}$ & $\begin{array}{l}0,0 \\
0,3\end{array}$ & $\begin{array}{l}0,0 \\
0,0\end{array}$ & $\begin{array}{l}1,0 \\
0,2\end{array}$ & $\begin{array}{r}28,0 \\
4,2\end{array}$ & $\begin{array}{l}47,6 \\
59,7\end{array}$ & $\begin{array}{l}11,5 \\
40,0\end{array}$ & $\begin{array}{l}2,5 \\
8,0\end{array}$ & $\begin{array}{l}1,2 \\
2,8\end{array}$ & $\begin{array}{l}0,4 \\
1,9\end{array}$ & $\begin{array}{l}0,2 \\
0,5\end{array}$ & $\begin{array}{l}1,8 \\
0,3\end{array}$ & $\begin{array}{l}0,4 \\
0,3\end{array}$ & $\begin{array}{l}19,9 \\
28,2\end{array}$ \\
\hline Palmae & $\begin{array}{l}1992 \\
1993\end{array}$ & $\begin{array}{l}6,3 \\
3,4\end{array}$ & $\begin{array}{l}0,2 \\
0,0\end{array}$ & $\begin{array}{l}0,1 \\
0,1\end{array}$ & $\begin{array}{l}0,1 \\
0,1\end{array}$ & $\begin{array}{l}0,0 \\
0,0\end{array}$ & $\begin{array}{l}0,0 \\
0,0\end{array}$ & $\begin{array}{l}2,5 \\
0,4\end{array}$ & $\begin{array}{l}3,4 \\
1,1\end{array}$ & $\begin{array}{l}9,3 \\
1,4\end{array}$ & $\begin{array}{l}4,8 \\
0,2\end{array}$ & $\begin{array}{r}4,3 \\
15,9\end{array}$ & $\begin{array}{l}3,5 \\
0,8\end{array}$ & $\begin{array}{l}0,8 \\
0,3\end{array}$ \\
\hline Parkinsonia & $\begin{array}{l}1992 \\
1993\end{array}$ & $\begin{array}{l}0,0 \\
0,0\end{array}$ & $\begin{array}{l}0,0 \\
0,0\end{array}$ & $\begin{array}{l}0,0 \\
0,0\end{array}$ & $\begin{array}{l}0,1 \\
0,0\end{array}$ & $\begin{array}{l}0,0 \\
0,0\end{array}$ & $\begin{array}{l}3,0 \\
0,7\end{array}$ & $\begin{array}{l}1,8 \\
2,0\end{array}$ & $\begin{array}{l}2,4 \\
0,9\end{array}$ & $\begin{array}{l}0,2 \\
0,9\end{array}$ & $\begin{array}{l}0,2 \\
0,0\end{array}$ & $\begin{array}{l}0,0 \\
0,0\end{array}$ & $\begin{array}{l}0,0 \\
0,0\end{array}$ & $\begin{array}{l}0,3 \\
0,2\end{array}$ \\
\hline Pinus & $\begin{array}{l}1992 \\
1993\end{array}$ & $\begin{array}{l}0,0 \\
0,0\end{array}$ & $\begin{array}{l}2,3 \\
1,2\end{array}$ & $\begin{array}{l}13,2 \\
14,3\end{array}$ & $\begin{array}{l}2,3 \\
4,4\end{array}$ & $\begin{array}{l}0,8 \\
1,1\end{array}$ & $\begin{array}{l}1,0 \\
6,7\end{array}$ & $\begin{array}{l}4,4 \\
2,4\end{array}$ & $\begin{array}{l}0,6 \\
1,3\end{array}$ & $\begin{array}{l}0,2 \\
0,7\end{array}$ & $\begin{array}{l}0,2 \\
0,1\end{array}$ & $\begin{array}{l}0,0 \\
0,0\end{array}$ & $\begin{array}{l}0,0 \\
0,0\end{array}$ & $\begin{array}{l}2,8 \\
4,1\end{array}$ \\
\hline Pistacia & $\begin{array}{l}1992 \\
1993\end{array}$ & $\begin{array}{l}0,0 \\
0,0\end{array}$ & $\begin{array}{l}0,0 \\
0,0\end{array}$ & $\begin{array}{l}1,5 \\
0,6\end{array}$ & $\begin{array}{l}0,8 \\
1,4\end{array}$ & $\begin{array}{l}0,0 \\
0,0\end{array}$ & $\begin{array}{l}0,0 \\
0,0\end{array}$ & $\begin{array}{l}0,0 \\
0,0\end{array}$ & $\begin{array}{l}0,0 \\
0,0\end{array}$ & $\begin{array}{l}0,0 \\
0,0\end{array}$ & $\begin{array}{l}0,0 \\
0,0\end{array}$ & $\begin{array}{l}0,0 \\
0,0\end{array}$ & $\begin{array}{l}0,0 \\
0,0\end{array}$ & $\begin{array}{l}0,3 \\
0,3\end{array}$ \\
\hline Plantago & $\begin{array}{l}1992 \\
1993\end{array}$ & $\begin{array}{l}0,0 \\
0,3\end{array}$ & $\begin{array}{l}0,0 \\
0,1\end{array}$ & $\begin{array}{l}4,2 \\
1,8\end{array}$ & $\begin{array}{r}11,6 \\
9,4\end{array}$ & $\begin{array}{l}4,8 \\
6,2\end{array}$ & $\begin{array}{l}5,3 \\
4,0\end{array}$ & $\begin{array}{l}8,3 \\
3,7\end{array}$ & $\begin{array}{l}2,0 \\
2,1\end{array}$ & $\begin{array}{l}1,3 \\
0,9\end{array}$ & $\begin{array}{l}1,0 \\
0,0\end{array}$ & $\begin{array}{l}0,3 \\
0,3\end{array}$ & $\begin{array}{l}0,2 \\
0,0\end{array}$ & $\begin{array}{l}4,9 \\
4,8\end{array}$ \\
\hline Platanus & $\begin{array}{l}1992 \\
1993\end{array}$ & $\begin{array}{l}0,0 \\
0,0\end{array}$ & $\begin{array}{l}0,0 \\
0,0\end{array}$ & $\begin{array}{l}7,4 \\
7,8\end{array}$ & $\begin{array}{l}0,3 \\
0,6\end{array}$ & $\begin{array}{l}0,0 \\
0,0\end{array}$ & $\begin{array}{l}0,0 \\
0,0\end{array}$ & $\begin{array}{l}0,0 \\
0,0\end{array}$ & $\begin{array}{l}0,0 \\
0,0\end{array}$ & $\begin{array}{l}0,0 \\
0,0\end{array}$ & $\begin{array}{l}0,0 \\
0,0\end{array}$ & $\begin{array}{l}0,0 \\
0,0\end{array}$ & $\begin{array}{l}0,0 \\
0,0\end{array}$ & $\begin{array}{l}0,9 \\
0,9\end{array}$ \\
\hline Populus & $\begin{array}{l}1992 \\
1993\end{array}$ & $\begin{array}{l}0,0 \\
0,0\end{array}$ & $\begin{array}{l}1,0 \\
0,4\end{array}$ & $\begin{array}{l}3,1 \\
0,8\end{array}$ & $\begin{array}{l}0,0 \\
0,0\end{array}$ & $\begin{array}{l}0,0 \\
0,0\end{array}$ & $\begin{array}{l}0,0 \\
0,0\end{array}$ & $\begin{array}{l}0,0 \\
0,0\end{array}$ & $\begin{array}{l}0,0 \\
0,0\end{array}$ & $\begin{array}{l}0,0 \\
0,0\end{array}$ & $\begin{array}{l}0,0 \\
0,0\end{array}$ & $\begin{array}{l}0,0 \\
0,0\end{array}$ & $\begin{array}{l}0,0 \\
0,0\end{array}$ & $\begin{array}{l}0,5 \\
0,1\end{array}$ \\
\hline Quercus & $\begin{array}{l}1992 \\
1993\end{array}$ & $\begin{array}{l}0,0 \\
0,7\end{array}$ & $\begin{array}{l}0,0 \\
0,0\end{array}$ & $\begin{array}{l}8,2 \\
1,5\end{array}$ & $\begin{array}{l}19,6 \\
38,6\end{array}$ & $\begin{array}{r}11,8 \\
4,5\end{array}$ & $\begin{array}{l}4,6 \\
6,1\end{array}$ & $\begin{array}{l}1,4 \\
1,9\end{array}$ & $\begin{array}{l}0,4 \\
0,9\end{array}$ & $\begin{array}{l}0,2 \\
1,6\end{array}$ & $\begin{array}{l}0,4 \\
0,6\end{array}$ & $\begin{array}{l}0,3 \\
0,3\end{array}$ & $\begin{array}{l}0,6 \\
0,5\end{array}$ & $\begin{array}{l}8,5 \\
9,7\end{array}$ \\
\hline Ricinus & $\begin{array}{l}1992 \\
1993\end{array}$ & $\begin{array}{l}6,8 \\
1,4\end{array}$ & $\begin{array}{l}0,2 \\
0,1\end{array}$ & $\begin{array}{l}0,6 \\
0,1\end{array}$ & $\begin{array}{l}0,4 \\
0,1\end{array}$ & $\begin{array}{l}0,4 \\
0,1\end{array}$ & $\begin{array}{l}0,3 \\
0,0\end{array}$ & $\begin{array}{l}1,0 \\
0,1\end{array}$ & $\begin{array}{l}1,4 \\
0,4\end{array}$ & $\begin{array}{l}0,4 \\
0,7\end{array}$ & $\begin{array}{l}0,4 \\
0,1\end{array}$ & $\begin{array}{l}3,1 \\
1,2\end{array}$ & $\begin{array}{l}2,3 \\
1,6\end{array}$ & $\begin{array}{l}0,6 \\
0,1\end{array}$ \\
\hline Rumex & $\begin{array}{l}1992 \\
1993\end{array}$ & $\begin{array}{l}0,3 \\
0,7\end{array}$ & $\begin{array}{l}0,0 \\
0,1\end{array}$ & $\begin{array}{l}1,7 \\
0,8\end{array}$ & $\begin{array}{l}2,6 \\
2,0\end{array}$ & $\begin{array}{l}1,3 \\
1,3\end{array}$ & $\begin{array}{l}1,5 \\
1,2\end{array}$ & $\begin{array}{l}1,0 \\
1,0\end{array}$ & $\begin{array}{l}0,2 \\
0,2\end{array}$ & $\begin{array}{l}0,0 \\
0,2\end{array}$ & $\begin{array}{l}0,0 \\
0,0\end{array}$ & $\begin{array}{l}0,3 \\
0,0\end{array}$ & $\begin{array}{l}0,2 \\
0,0\end{array}$ & $\begin{array}{l}1,2 \\
1,2\end{array}$ \\
\hline Typha & $\begin{array}{l}1992 \\
1993\end{array}$ & $\begin{array}{l}0,0 \\
0,0\end{array}$ & $\begin{array}{l}0,0 \\
0,0\end{array}$ & $\begin{array}{l}0,0 \\
0,0\end{array}$ & $\begin{array}{l}0,0 \\
0,0\end{array}$ & $\begin{array}{l}0,2 \\
0,0\end{array}$ & $\begin{array}{l}2,6 \\
0,6\end{array}$ & $\begin{array}{l}0,4 \\
0,4\end{array}$ & $\begin{array}{l}0,2 \\
0,4\end{array}$ & $\begin{array}{l}0,2 \\
0,2\end{array}$ & $\begin{array}{l}0,0 \\
0,0\end{array}$ & $\begin{array}{l}0,0 \\
0,0\end{array}$ & $\begin{array}{l}0,0 \\
0,0\end{array}$ & $\begin{array}{l}0,2 \\
0,1\end{array}$ \\
\hline Ulmus & $\begin{array}{l}1992 \\
1993\end{array}$ & $\begin{array}{l}0,3 \\
0,0\end{array}$ & $\begin{array}{l}0,2 \\
0,2\end{array}$ & $\begin{array}{l}0,1 \\
0,2\end{array}$ & $\begin{array}{l}0,0 \\
0,0\end{array}$ & $\begin{array}{l}0,0 \\
0,0\end{array}$ & $\begin{array}{l}0,0 \\
0,0\end{array}$ & $\begin{array}{l}0,0 \\
0,0\end{array}$ & $\begin{array}{l}0,0 \\
0,0\end{array}$ & $\begin{array}{l}0,0 \\
0,0\end{array}$ & $\begin{array}{l}0,0 \\
0,0\end{array}$ & $\begin{array}{l}0,0 \\
0,0\end{array}$ & $\begin{array}{l}0,0 \\
0,0\end{array}$ & $\begin{array}{l}0,0 \\
0,0\end{array}$ \\
\hline Urticaceae & $\begin{array}{l}1992 \\
1993 \\
\end{array}$ & $\begin{array}{l}12,1 \\
21,4 \\
\end{array}$ & $\begin{array}{l}2,3 \\
2,9 \\
\end{array}$ & $\begin{array}{l}7,5 \\
6,8 \\
\end{array}$ & $\begin{array}{l}4,7 \\
4,8 \\
\end{array}$ & $\begin{array}{l}2,0 \\
1,9 \\
\end{array}$ & $\begin{array}{l}5,9 \\
2,3 \\
\end{array}$ & $\begin{array}{l}7,8 \\
4,3 \\
\end{array}$ & $\begin{array}{l}1,8 \\
3,6 \\
\end{array}$ & $\begin{array}{l}0,6 \\
1,6 \\
\end{array}$ & $\begin{array}{l}1,4 \\
0,6 \\
\end{array}$ & $\begin{array}{l}10,9 \\
19,3 \\
\end{array}$ & $\begin{array}{r}6,8 \\
35,2 \\
\end{array}$ & $\begin{array}{l}4,0 \\
3,9 \\
\end{array}$ \\
\hline Suma & \begin{tabular}{|l|}
1992 \\
1993 \\
\end{tabular} & $\begin{array}{l}77,4 \\
87,4\end{array}$ & $\begin{array}{l}97,9 \\
98,2\end{array}$ & $\begin{array}{l}82,3 \\
94,2\end{array}$ & $\begin{array}{l}88,6 \\
84,9\end{array}$ & $\begin{array}{l}90,3 \\
93,5\end{array}$ & $\begin{array}{l}89,0 \\
85,1\end{array}$ & $\begin{array}{l}79,9 \\
76,8 \\
\end{array}$ & $\begin{array}{l}72,7 \\
70,2\end{array}$ & $\begin{array}{l}86,2 \\
73,5\end{array}$ & $\begin{array}{l}86,0 \\
91,0\end{array}$ & $\begin{array}{l}86,5 \\
87,3\end{array}$ & $\begin{array}{l}90,7 \\
77,3\end{array}$ & $\begin{array}{l}89,2 \\
89,3\end{array}$ \\
\hline
\end{tabular}

Tabla 3. Porcentaje mensual y anual de los taxones estudiados con respecto al polen total obtenido durante los años 1992 y 1993. Monthly and annual percentage of the pollen types studied in relation with the total pollen obtained during 1992 and 1993. 


\begin{tabular}{|c|c|c|c|c|c|}
\hline Taxón & Año & Día pico & $\begin{array}{c}\text { Concentración } \\
\text { media }\end{array}$ & Semana pico & $\begin{array}{c}\text { Concentración } \\
\text { media }\end{array}$ \\
\hline Cupressaceae & $\begin{array}{l}1992 \\
1993\end{array}$ & $\begin{array}{l}17 \text {-febrero } \\
22 \text {-febrero }\end{array}$ & $\begin{array}{l}435 \\
414\end{array}$ & $\begin{array}{l}2^{a} \text { febrero } \\
4^{a} \text { febrero }\end{array}$ & $\begin{array}{l}220 \\
141\end{array}$ \\
\hline Pinus & $\begin{array}{l}1992 \\
1993\end{array}$ & $\begin{array}{l}\text { 17-junio } \\
\text { 8-marzo }\end{array}$ & $\begin{array}{c}93 \\
176\end{array}$ & $\begin{array}{c}3^{\mathrm{a}} \text { marzo } \\
3^{\mathrm{a}} \text { junio }\end{array}$ & $\begin{array}{l}14 \\
42\end{array}$ \\
\hline Platanus & $\begin{array}{l}1992 \\
1993\end{array}$ & $\begin{array}{l}\text { 14-marzo } \\
\text { 21-marzo }\end{array}$ & $\begin{array}{l}59 \\
43\end{array}$ & $\begin{array}{l}2^{\mathrm{a}} \text { marzo } \\
3^{\mathrm{a}} \text { marzo }\end{array}$ & $\begin{array}{l}13 \\
17\end{array}$ \\
\hline Urticaceae & $\begin{array}{l}1992 \\
1993\end{array}$ & $\begin{array}{c}\text { 18-marzo } \\
\text { 7-abril }\end{array}$ & $\begin{array}{l}17 \\
29\end{array}$ & $\begin{array}{c}3^{\mathrm{a}} \text { marzo } \\
1^{\mathrm{a}} \text { abril }\end{array}$ & $\begin{array}{c}9 \\
19\end{array}$ \\
\hline Quercus & $\begin{array}{l}1992 \\
1993\end{array}$ & $\begin{array}{c}\text { 10-mayo } \\
9 \text {-abril }\end{array}$ & $\begin{array}{l}125 \\
346\end{array}$ & $\begin{array}{c}2^{\mathrm{a}} \text { mayo } \\
1^{\mathrm{a}} \text { abril }\end{array}$ & $\begin{array}{l}43 \\
126\end{array}$ \\
\hline Olea europaea & $\begin{array}{l}1992 \\
1993\end{array}$ & $\begin{array}{l}\text { 2-mayo } \\
\text { 9-mayo }\end{array}$ & $\begin{array}{l}627 \\
745\end{array}$ & $\begin{array}{l}2^{\mathrm{a}} \text { mayo } \\
2^{\mathrm{a}} \text { mayo }\end{array}$ & $\begin{array}{l}153 \\
352\end{array}$ \\
\hline Plantago & $\begin{array}{l}1992 \\
1993\end{array}$ & $\begin{array}{c}\text { 16-abril } \\
15 \text {-mayo }\end{array}$ & $\begin{array}{l}56 \\
68\end{array}$ & $\begin{array}{c}3^{\mathrm{a}} \text { abril } \\
2^{\mathrm{a}} \text { mayo }\end{array}$ & $\begin{array}{l}26 \\
32\end{array}$ \\
\hline Chenop.-Amar. & $\begin{array}{l}1992 \\
1993\end{array}$ & $\begin{array}{l}\text { 29-abril } \\
23 \text {-mayo }\end{array}$ & $\begin{array}{c}60 \\
107\end{array}$ & $\begin{array}{c}4^{\mathrm{a}} \text { abril } \\
1^{\mathrm{a}} \text { mayo }\end{array}$ & $\begin{array}{l}41 \\
52\end{array}$ \\
\hline Gramineae & $\begin{array}{l}1992 \\
1993\end{array}$ & $\begin{array}{l}\text { 11-mayo } \\
2 \text {-junio }\end{array}$ & $\begin{array}{l}99 \\
60\end{array}$ & $\begin{array}{l}2^{\mathrm{a}} \text { mayo } \\
1^{\mathrm{a}} \text { junio }\end{array}$ & $\begin{array}{l}28 \\
44\end{array}$ \\
\hline Myrtaceae & $\begin{array}{l}1992 \\
1993\end{array}$ & $\begin{array}{c}\text { 1-julio } \\
22 \text {-junio }\end{array}$ & $\begin{array}{l}40 \\
31\end{array}$ & $\begin{array}{l}4^{\mathrm{a}} \text { junio } \\
2^{\mathrm{a}} \text { julio }\end{array}$ & $\begin{array}{l}17 \\
13\end{array}$ \\
\hline Casuarina & $\begin{array}{l}1992 \\
1993\end{array}$ & $\begin{array}{l}27 \text {-octubre } \\
22 \text {-octubre }\end{array}$ & $\begin{array}{l}25 \\
166\end{array}$ & $\begin{array}{l}4^{\mathrm{a}} \text { octubre } \\
3^{\mathrm{a}} \text { octubre }\end{array}$ & $\begin{array}{l}15 \\
64\end{array}$ \\
\hline Artemisia & $\begin{array}{l}1992 \\
1993\end{array}$ & $\begin{array}{l}\text { 20-diciembre } \\
\text { 12-diciembre }\end{array}$ & $\begin{array}{l}51 \\
17\end{array}$ & $\begin{array}{l}3^{\mathrm{a}} \text { diciembre } \\
2^{\mathrm{a}} \text { diciembre }\end{array}$ & $\begin{array}{c}24 \\
4\end{array}$ \\
\hline
\end{tabular}

Tabla 4. Valores máximos obtenidos por los 12 taxones más importantes en la atmósfera de Málaga durante los años 1992 y 1993 (día pico y semana pico). Maximum values obtained by the 12 more important taxa in the atmosphere of Malaga during 1992 and 1993 (day peak and week peak).

na. Por otra parte, Junio se caracteriza por el aumento del polen de taxones típicamente estivales como son las Mirtáceas (fundamentalmente Eucalyptus), Umbelíferas, Castanea y Typha. Existen grandes diferencias entre las cantidades de polen recogidas durante este mismo mes en los dos años objetos de estudio, pasándose de 1402 granos de polen total en 1992 a 6219 en 1993, aunque probablemente estas diferencias sean debidas a las precipitaciones que durante buena parte del mes de junio hicieron descender las concentraciones de 1992.

Durante Julio, Agosto y Septiembre, de- bido a la ausencia de precipitaciones y al aumento de las temperaturas, las concentraciones polínicas se mantienen en niveles muy bajos, sin que ninguna especie alcance concentraciones importantes, siendo Quenopodiáceas, Gramíneas y Mirtáceas los taxones con mayor importancia relativa.

Hacia mediados de Octubre se produce un nuevo incremento de las concentraciones polínicas debido fundamentalmente a la aparición de polen de Casuarina, que representó durante este mes el 41,1\% del total en 1992 y el 78,6\% en 1993 y, aunque no suele alcanzar valores extremadamente altos, hay que tener 


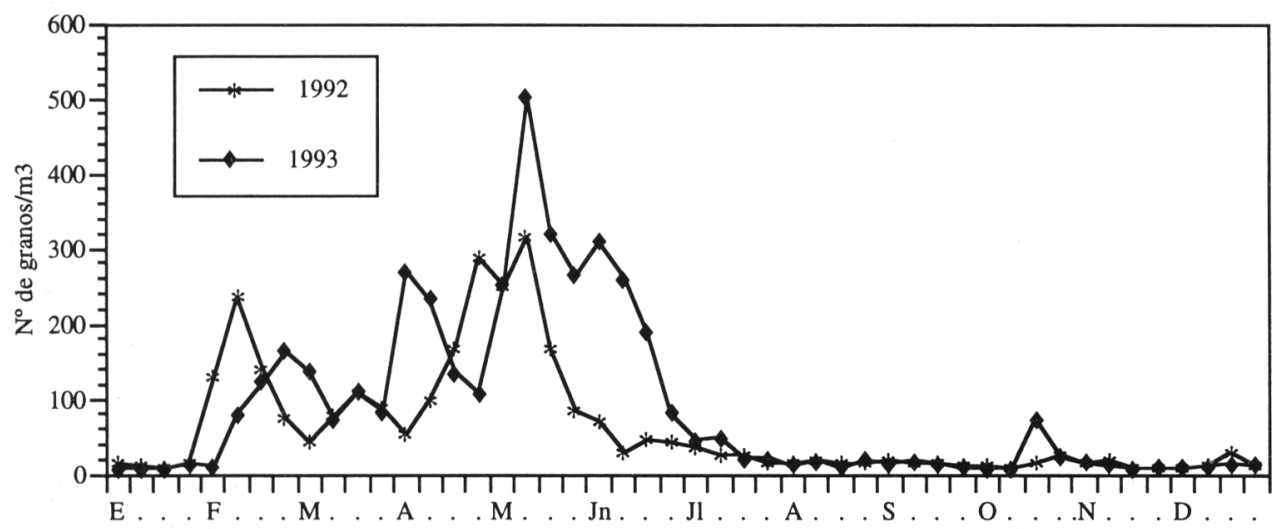

Figura 1: Evolución de la concentracion media semanal del polen total en la atmósfera de Málaga durante los años 1992 y 1993. Total pollen concentration trend in the atmosphere of Málaga during 1992 and 1993 (weekly average).

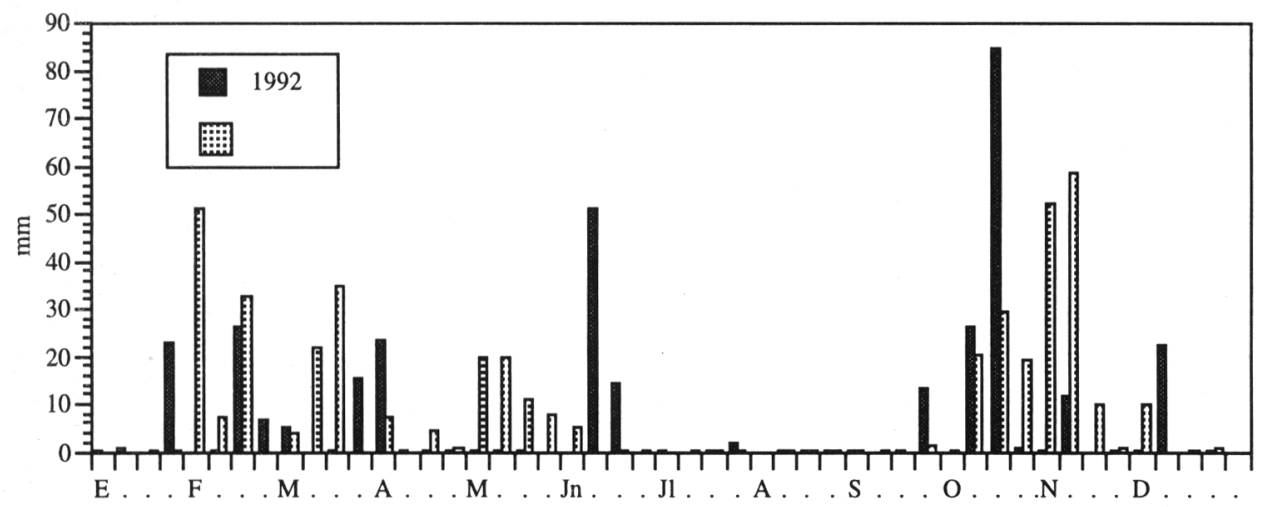

Figura 2: Precipitaciones totales semanales registradas en Málaga durante los años 1992 y 1993 . Weekly total rainfalls in Málaga during 1992 and 1993.

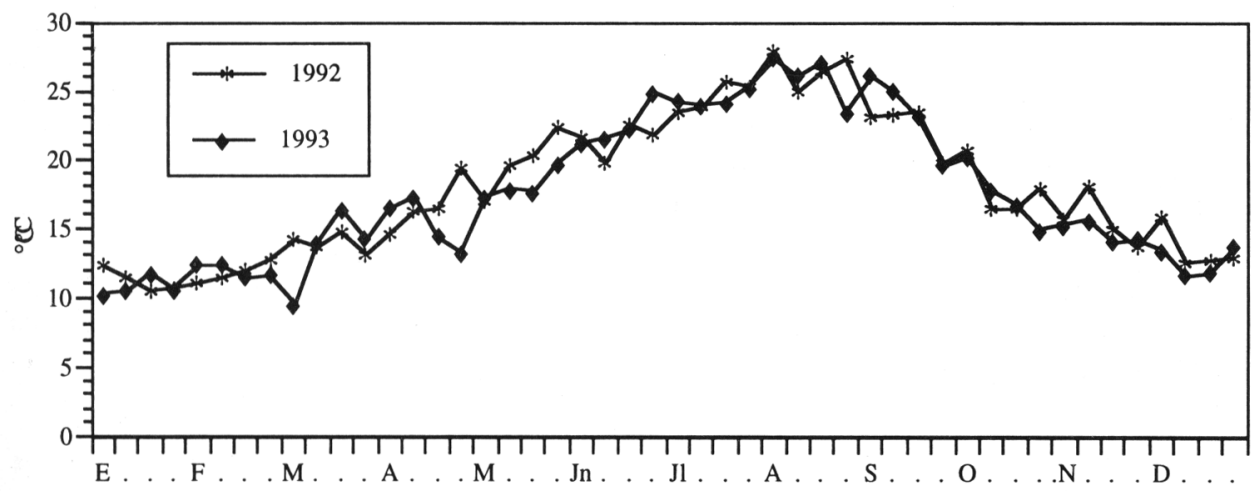

Figura 3: Evolución de la temperatura media semanal durante los años 1992 y 1993 . Weekly mean temperature trend in Málaga during 1992 and 1993. 
en cuenta, dado el carácter alergénico que presenta (Bucholtz et al., 1987), que se trata de una especie que aparece en un momento en el que la atmósfera está prácticamente libre de otros tipos de polen.

Noviembre y Diciembre, junto con Enero, son los meses en que las concentraciones de polen se mantienen en valores más bajos, en los que sólo cabe destacar la presencia de polen de Artemisia, Cupresáceas y Urticáceas.

En general, puede decirse que las grandes diferencias observadas en las cantidades de polen total recogidas un año con respecto al otro, vienen determinadas a su vez por diferencias observadas en las concentraciones de los taxones que mayor incidencia tienen en la atmósfera. Así, se observa que los totales obtenidos durante 1993 fueron sensiblemente superiores a los de 1992. Esta diferencia fue debida a un fuerte incremento de pólenes procedentes de especies arbóreas, fundamentalmente de olivo y Quercus, pero también, aunque en menor proporción, de Pinus, Casuarina y Platanus. También son de destacar las mayores concentraciones obtenidas durante ese mismo año por los pólenes de QuenopodiáceasAmarantáceas, mientras que las Cupresáceas, sin embargo, se mantuvieron en niveles similares.

Por último, con objeto de dar una idea de los valores máximos alcanzados por los diferentes tipos polínicos estudiados, así como los desfases ocurridos de un año a otro, en la tabla 4 se indican cuáles fueron los días y las semanas de máxima concentración de polen de los 12 taxones más importantes durante los dos años objeto de estudio.

\section{CONCLUSIONES}

Durante los años 1992 y 1993 alrededor del $85 \%$ del polen total se obtuvo durante los meses de Febrero a Junio, ambos inclusive, siendo Mayo el mes en que se registran los picos de máxina concentración.

Los valores mensuales más bajos de polen total se obtienen durante los meses de Agosto a Diciembre, así como durante el mes de Enero.

Los taxones que mayor incidencia tuvieron en la atmósfera de Málaga durante 1992 y 1993 fueron Cupresáceas y olivo, seguidos, en orden de importancia por Quercus, QuenopodiáceasAmarantáceas, Gramíneas, Plantago, Urticáceas y Pinus.

Los distintos picos que se sucedieron a lo largo de los años de estudio se deben fundamentalmente a Cupresáceas (Febrero), Cupresáceas-PinusPatanus (Marzo), Quercus (Abril), OlivoQuenopodiáceas-Gramíneas (Mayo) y Casuarina (Octubre).

AGRADECIMIENTOS. Los autores desean agradecer la colaboración prestada a la Red Andaluza de Aerobiología (RAA) por las Consejerías de Salud y Educación de la Junta de Andalucía, y por el Ilustre Colegio Oficial de Farmacéuticos de Málaga.

\section{BIBLIOGRAFÍA}

BUCHOLTZ, G. A., A.E. HENSEL, R.F. LOCKEY, D. SERBOUSEK \& R. P. WUNDERLIN - 1987 Australian pine (Casuarina equisetifolia) pollen as an aeroallergen. Annals of Allergy, 59: 52-56.

BURGOS, F. -1991- Pólenes y medio ambiente: sensibilizaciones polínicas en Andalucía. Actas de la XX Reunión de la Asociación de Alergólogos e Inmunólogos del Sur (AISUR): 134-141, Huelva.

CHAPARRO, A. y J. CONDE -1984- Estudio y comentarios de las estaciones mediterráneas costeras. Alergia a pólenes: 44-50. I Ponencia desarrollada en el XIV Congreso de la Sociedad Española de Alergia e Inmunología Clínica, Santiago de Compostela.

D’AMATO, G. A. \& F. TH. M. SPIEKSMA -1990Allergenic Pollen in Europe. Grana, 30: 67-70.

DOMÍNGUEZ VILCHES, E., C. GALÁN SOLDEVILLA, F. VILLAMANDOS DE LA TORRE \& F. INFANTE GARCÍAPANTALEÓN - 1991- Handling and evaluation of the data from the aerobiological sampling. Monografías REA/EAN 1: 1-18.

Aceptado para su publicación en Julio de 1994

Dirección de los autores. Departamento de Biología Vegetal. Facultad de Ciencias. Universidad de Málaga. Apdo. 59. 29080, Málaga. Fax (95) 2131944. 